\title{
Mona Hoppenrath \\ A revised checklist of planktonic diatoms and dinoflagellates from Helgoland (North Sea, German Bight)
}

\author{
Received: 13 May 2003/ Revised: 11 April 2004 / Accepted: 10 July 2004 / Published online: 20 October 2004
}

(c) Springer-Verlag and AWI 2004

\begin{abstract}
A checklist based on net samples taken twice weekly from 2001 until May 2003 is presented. Identification is based on observations under direct light microscopy and after taking some organisms in culture. The checklist includes 227 taxa observed at the Helgoland Reede sampling station. One hundred and thirtytwo species of diatoms from 53 genera and 95 species of dinoflagellates from 35 genera have been recorded from net samples and cultures. Thirty-five diatom and 28 dinoflagellate taxa were documented in the Helgoland phytoplankton for the first time. The list does not claim to be complete, but provides an updated list of the taxa found at Helgoland and, for convenience, also includes data published for different adjacent areas.
\end{abstract}

Keywords Diatoms - Dinoflagellates - Species list . Helgoland Reede

\section{Introduction}

Phytoplankton species composition and physico-chemical parameters have been recorded in the framework of a time series at Helgoland since 1962 (Hagmeier et al. 2004; Van Beusekom et al. 2004; Wiltshire and Dürselen 2004; Wiltshire et al. 2004). The Helgoland Reede station is sampled daily on weekdays. Water-bottle samples were fixed and phytoplankton taxa counted mainly for the estimation of the carbon content (quantification for the determination of biomass).

Communicated by K. Wiltshire

M. Hoppenrath

Biologische Anstalt Helgoland, Alfred Wegener Institute

Foundation for Polar and Marine Research,

Kurpromenade, 27498 Helgoland, Germany

E-mail: mhoppenrath@awi-bremerhaven.de

Tel.: + 49-4725-819255

Fax: $+49-4725-819283$
Since the species survey by Drebes from 1966 to 1971 (Drebes 1974), there has been no continuous species composition record from net samples. For that reason there was no actual data set covering microphytoplankton species diversity. This knowledge is not only important for getting an idea about the percentage of species diversity coverage that is recorded in the Helgoland timeseries, but also because there are continuous species composition records for the Wadden Sea phytoplankton at Sylt, Germany. The data presented here are the first results of an intensive taxonomic phytoplankton reinvestigation. They could be used as a basis for phytoplankton count quality control and for the start of a continuous detailed species recording in the framework of the Helgoland time series. Since the publication of the 1976 list (Drebes and Elbrächter 1976), nomenclatural changes have been sufficiently numerous to warrant the present list, incorporating additions, synonyms and corrections. Only those species that have been personally observed by the author and/or by Drebes (Drebes 1974; Drebes and Elbrächter 1976) are listed. It is an addition to the checklist of benthic species found in the vicinity of the island of Helgoland (Harms 1993).

\section{Methods}

Net samples from the surface water of the Helgoland Reede station $\left(54^{\circ} 11.30^{\prime} \mathrm{N} 7^{\circ} 54.00^{\prime} \mathrm{E}\right)$ were collected twice a week from 2001 until May 2003 for qualitative species identification (in addition to the routine waterbottle samples for biomass determination). Nets of different mesh size, $20 \mu \mathrm{m}$ and $80 \mu \mathrm{m}$, were used. The samples were brought to the laboratory and living organisms in small Petri dishes were identified using an inverted light microscope equipped with seawaterimmersion objectives for about half a day. All species observed and identified were listed. Additionally, diatom cells were isolated and cultured and some samples were fixed with Lugol's solution, for further investigations 
Table 1 Checklist of marine phytoplankton, diatoms and dinoflagellates from Helgoland and other North Sea areas. 1 Hoppenrath, present study; 2 Drebes (1974); 3 Drebes and Elbrächter (1976); 4 Parke and Dixon (1976; coasts of Britain and Ireland and adjacent waters); 5 Hendey (1974; British marine diatoms); 6 Hartley (1986; marine diatoms of the British Isles and adjoining coastal waters);
7 Heimdal et al. (1973; Oslofjord, Norway); 8 Kuylenstierna and Karlson (2000; Skagerrak and Kattegat, Sweden); 9 Hansen and Larsen (1992; Kattegat, Denmark); 10 Leewis (1985; Dutch coast); 11 Pankow (1990; Baltic Sea); 12 Hällfors (Baltic Sea); $t$ potentially toxic species

Species

Listed in:

\begin{tabular}{llllllllllll}
\hline 1 & 2 & 3 & 4 & 5 & 6 & 7 & 8 & 9 & 10 & 11 & 12
\end{tabular}

\section{Bacillariophyceae}

Achnanthes longipes Agardh

Actinocyclus curvatulus Janisch (syn.

Coscinodiscus curvatulus)

Actinocyclus octonarius Ehrenberg (syn.

Actinocyclus ehrenbergii)

Actinoptychus senarius (Ehrenberg)

Ehrenberg (syn. Actinoptychus undulatus)

Actinoptychus splendens (Shadbbolt) Ralfs

Asterionellopsis glacialis (Castracane) Round

(syn. Asterionella glacialis, Asterionella japonica)

Asteroplanus karianus (Grunow) Crawford

(syn. Asterionella karianus)

Aulacodiscus argus (Ehrenberg) Schmidt

Bacillaria paxillifer (O.F. Müller) Hendey

(syn. Bacillaria paradoxa)

Bacteriastrum hyalinum Lauder

Bellerochea malleus (Brightwell) van Heurck

(syn. Triceratium malleus)

Biddulphia alternans (Bailey) van Heurck

(syn.Triceratium alternans)

Brockmanniella brockmannii (Hustedt) Hasle

et al. (syn. Plagiogramma brockmannii)

Campylosira cymbelliformis Van Heurck

Calyptrella robusta (Norman) Hernández-

Becerril et Meave (syn.Rhizosolenia robusta)

Catenula adhaerens (Mereschkowsky)

Mereschkowsky

Cerataulina pelagica (Cleve) Hendey

(syn.Cerataulina bergonii)

Cerataulus radiatus (Roper) Ross

(syn.Cerataulus smithii)

Chaetoceros affinis Lauder

Chaetoceros borealis Bailey

Chaetoceros compressus Lauder ${ }^{\text {a }}$

Chaetoceros convolutus Castracane

Chaetoceros coronatus Gran

Chaetoceros costatus Gran

Chaetoceros crinitus Schütt

Chaetoceros curvisetus Cleve

Chaetoceros danicus Cleve

Chaetoceros debilis Cleve

Chaetoceros decipiens Cleve

Chaetoceros densus (Cleve) Cleve

Chaetoceros diadema (Ehrenberg) Gran (syn.

Chaetoceros subsecundus)

Chaetoceros didymus Ehrenberg

Chaetoceros eibenii Grunow

Chaetoceros externus Gran

Chaetoceros lauderi Ralfs

Chaetoceros protuberans Lauder

Chaetoceros radicans Schütt

Chaetoceros similis Cleve

Chaetoceros simplex Ostenfeld

Chaetoceros socialis Lauder

Chaetoceros subtilis Cleve

Chaetoceros teres Cleve

Chaetoceros tortissimus Gran

Chaetoceros willei Gran ${ }^{\mathrm{b}}$

Corethron hystrix Hensen

\begin{tabular}{|c|c|c|c|c|c|c|c|c|c|}
\hline $\begin{array}{l}+ \\
+\end{array}$ & & + & $\begin{array}{l}+ \\
?\end{array}$ & + & + & + & & + & + \\
\hline+ & + & + & + & + & & + & & + & + \\
\hline+ & + & + & + & + & & + & + & + & + \\
\hline+ & + & + & + & + & & & + & & + \\
\hline+ & + & + & + & + & + & + & + & + & + \\
\hline+ & + & + & + & + & & + & + & & + \\
\hline+ & + & + & + & + & & & + & & + \\
\hline+ & + & & + & + & + & & + & + & + \\
\hline+ & + & + & + & + & & + & + & & + \\
\hline+ & + & + & + & + & & & + & & + \\
\hline+ & + & + & + & + & & & + & & \\
\hline+ & + & + & + & + & & & + & & \\
\hline & & + & & + & & & & & \\
\hline+ & + & + & + & + & & & + & & + \\
\hline+ & & & + & + & & & & + & + \\
\hline+ & + & + & + & + & + & + & + & + & + \\
\hline+ & + & + & + & + & & & + & & + \\
\hline+ & + & + & & + & + & + & & + & + \\
\hline+ & + & + & + & + & + & + & + & + & + \\
\hline $\mathrm{cf}+$ & + & + & + & + & + & + & + & + & + \\
\hline & & + & + & + & + & + & & & + \\
\hline & & + & + & + & & + & & + & + \\
\hline+ & + & + & + & + & & + & & & + \\
\hline $\mathrm{cf}+$ & + & + & + & + & & & & + & + \\
\hline+ & + & + & + & & + & + & + & + & + \\
\hline+ & + & + & + & + & + & + & + & + & + \\
\hline+ & + & + & + & + & + & + & + & + & + \\
\hline+ & + & + & + & + & + & + & & + & + \\
\hline+ & + & + & + & + & & + & + & + & + \\
\hline+ & + & + & + & + & & + & & + & + \\
\hline+ & + & + & + & + & + & + & + & + & + \\
\hline+ & + & + & + & + & & + & + & + & + \\
\hline & + & + & + & + & & & & & + \\
\hline+ & + & + & + & + & + & + & & + & + \\
\hline+ & + & + & & & & & & & + \\
\hline+ & + & + & & + & + & + & & & + \\
\hline+ & & & + & + & + & + & & + & + \\
\hline+ & & & + & + & + & + & + & + & + \\
\hline+ & + & + & + & + & + & + & + & + & + \\
\hline+ & & & + & + & + & + & + & + & + \\
\hline+ & + & + & + & + & + & + & + & + & + \\
\hline+ & + & + & + & + & & + & + & & + \\
\hline & + & + & + & + & & & & & \\
\hline+ & & & + & + & + & $\mathrm{cf}+$ & + & & \\
\hline
\end{tabular}


Table 1 (Contd.)

Species

Listed in:

\begin{tabular}{llllllllllll}
\hline 1 & 2 & 3 & 4 & 5 & 6 & 7 & 8 & 9 & 10 & 11 & 12 \\
\hline
\end{tabular}

Coscinodiscus commutatus Grunow (today:

Coscinodiscus centralis Ehrenberg?) ${ }^{\mathrm{c}}$

Coscinodiscus concinnus Smith

Coscinodiscus granii Gough

Coscinodiscus pavillardii Forti ${ }^{\mathrm{d}}$

Coscinodiscus radiatus Ehrenberg

Coscinodiscus stellaris Roper

Coscinodiscus wailesii Gran et Angst ${ }^{\mathrm{e}}$

Cylindrotheca closterium (Ehrenberg) Reimann

et Lewin (syn. Nitzschia closterium)

Delphineis surirella (Ehrenberg) Andrews

(syn.Rhaphoneis surirella)

Detonula confervacea (Cleve) Gran

Detonula pumila (Castracane) Schütt (syn.

Schroederella pumila, Schroederella schroederi)

Ditylum brightwellii (West) Grunow

Eucampia zodiacus Ehrenberg

Eunotogramma dubium Hustedt

Fragilaria islandica Grunow

Fragilaria oblonga Drebes et Schulz

Grammatophora marina (Lyngbye) Kützing

Guinardia delicatula (Cleve) Hasle (syn.

Rhizosolenia delicatula)

Guinardia flaccida (Castracane) Peragallo

Guinardia striata (Stolterfoth) Hasle (syn.

Rhizosolenia stolterfothii)

Helicothe catamesis (Shrubsole) Ricard

(syn. Streptothe catamesis)

Lauderia annulata Cleve (syn.Lauderia borealis)

Leptocylindrus danicus Cleve

Leptocylindrus minimus Gran

Lithodesmium undulatum Ehrenberg

Melosira moniliformis (O.F. Müller) Agardh

(syn. Melosira borreri)

Melosira nummuloides (Dillwyn) Agardh

Minidiscus trioculatus (Taylor) Hasle

(syn. Coscinodiscus trioculatus)

Nitzschia longissima (Kützing) Grunow

Odontella aurita (Lyngbye) Agardh

(syn. Biddulphia aurita)

Odontella aurita var. minima (Grunow) Drebes

Odontella granulata (Roper) Ross

(syn. Biddulphia granulata)

Odontella mobiliensis (Bailey) Grunow

(syn. Biddulphia mobiliensis)

Odontella obtusa Kützing

Odontella regia (Schultze) Simonsen

(syn. Biddulphia regia)

Odontella rhombus (Ehrenberg) Kützing

(syn. Biddulphia rhombus)

Odontella rhombus f. trigona (van Heurck) Ross

Odontella sinensis (Greville) Grunow

(syn. Biddulphia sinensis) ${ }^{\mathrm{f}}$

Paralia marina (Smith) Heiberg

(syn. Paralia sulcata)

Plagiogrammopsis vanheurckii (Grunow)

Hasle et al. (syn. Plagiogramma vanheurckii)

Podosira stelliger (Bailey) Mann

(syn. Hyalodiscus stelliger)

Porosira glacialis (Grunow) Jörgensen

(syn. Lauderia glacialis)

Proboscia alata (Brightwell) Sundström

(syn. Rhizosolenia alata)

Proboscia indica (Peragallo) Drebes

(syn.Rhizosolenia indica) $^{\mathrm{g}}$

\begin{tabular}{|c|c|c|c|c|c|c|c|c|}
\hline+ & + & + & & $?$ & $?$ & $?$ & $?$ & + \\
\hline+ & + & + & + & + & + & + & + & + \\
\hline+ & + & + & + & + & + & + & & + \\
\hline $\mathrm{cf}+$ & + & + & & $?$ & & & & \\
\hline+ & + & + & + & + & + & + & + & + \\
\hline & + & + & + & + & & & & \\
\hline+ & & & & + & & + & & \\
\hline+ & & & + & + & & + & $?$ & + \\
\hline+ & + & + & + & + & & & + & + \\
\hline+ & + & + & + & + & + & + & + & + \\
\hline+ & + & + & $?$ & & + & + & + & \\
\hline+ & + & + & + & + & + & + & + & + \\
\hline+ & + & + & + & + & + & + & + & + \\
\hline+ & + & $?+$ & + & + & & & & + \\
\hline+ & & & & & & & & \\
\hline $\mathrm{cf}+$ & & & + & + & & & & + \\
\hline+ & + & + & + & + & + & + & + & + \\
\hline+ & + & + & + & + & + & + & + & + \\
\hline+ & + & + & + & + & + & + & + & + \\
\hline+ & + & + & + & + & & + & + & \\
\hline+ & + & + & + & + & & + & + & + \\
\hline+ & + & + & + & + & + & + & + & + \\
\hline+ & + & + & + & + & & + & + & + \\
\hline+ & + & + & + & + & & + & + & + \\
\hline+ & + & + & + & + & + & & + & + \\
\hline $\begin{array}{l}+ \\
\mathrm{cf}+\end{array}$ & + & + & + & $\begin{array}{l}+ \\
+\end{array}$ & + & + & + & + \\
\hline & + & + & + & + & + & + & & + \\
\hline+ & + & + & + & + & + & + & + & + \\
\hline+ & & & & & & & + & \\
\hline+ & + & + & + & + & & & & \\
\hline+ & + & + & + & + & & + & + & + \\
\hline $\mathrm{cf}+$ & + & & + & + & & & & \\
\hline+ & + & + & + & + & & + & + & \\
\hline+ & + & + & + & + & & & + & \\
\hline+ & + & & + & + & & & & \\
\hline+ & + & + & + & + & + & + & + & + \\
\hline+ & + & + & + & + & & + & & + \\
\hline+ & & & + & + & & + & + & \\
\hline+ & + & + & + & + & + & & + & + \\
\hline+ & + & + & + & + & + & + & + & \\
\hline+ & + & + & + & + & + & + & & + \\
\hline+ & + & + & & $?$ & & & & \\
\hline
\end{tabular}


Table 1 (Contd.)

Species

Listed in:

\begin{tabular}{llllllllllll}
\hline 1 & 2 & 3 & 4 & 5 & 6 & 7 & 8 & 9 & 10 & 11 & 12 \\
\hline
\end{tabular}

Pseudo-nitzschia delicatissima (Cleve) Heiden

\begin{tabular}{|c|c|}
\hline $\mathrm{cf}+$ & + \\
\hline $\begin{array}{l}+ \\
+\end{array}$ & + \\
\hline+ & \\
\hline+ & + \\
\hline & + \\
\hline+ & + \\
\hline+ & + \\
\hline+ & + \\
\hline+ & \\
\hline+ & + \\
\hline+ & + \\
\hline+ & + \\
\hline+ & + \\
\hline+ & + \\
\hline+ & \\
\hline+ & \\
\hline+ & + \\
\hline $\begin{array}{l}+ \\
+\end{array}$ & \\
\hline+ & \\
\hline & + \\
\hline
\end{tabular}

$++$

(syn. Nitzschia delicatissima)

Pseudo-nitzschia fraudulenta Cleve $\mathbf{t}$

Pseudo-nitzschia pungens Grunow

[= Nitzschia "seriata" in Drebes (1974)] t

Pseudopodosira westii (Smith)

Sheshukova-Poretzkaya (syn. Melosira westii)

Rhaphoneis amphiceros (Ehrenberg) Ehrenberg

Rhizosolenia hebetata f. semispina (Hensen) Gran

Rhizosolenia imbricata Brightwell

(syn. Rhizosolenia shrubsolei)

Rhizosolenia pungens Cleve-Euler

Rhizosolenia setigera Brightwell

Rhizosolenia cf similoides Cleve-Euler

Rhizosolenia styliformis Brightwell

Roperiates selata (Roper) Grunow

Skeletonema costatum (Greville) Cleve

Stauropsis membranacea (Cleve) Meunier

(syn. Stauroneis membranacea)

Stephanopyxis turris (Greville et Arnott) Ralfs

Subsilicea fragilarioides Stosch et Reimann

Thalassionema frauenfeldii (Grunow) Hallegraeff

(syn. Thalassiothrix frauenfeldii)

Thalassionema nitzschioides (Grunow) Hustedt

Thalassiosira aestivalis Gran et Angst

Thalassiosira of allenii Takano

Thalassiosira angulata (Gregory) Hasle

Thalassiosira anguste-lineata (Schmidt) Fryxell et

Hasle (syn. Coscinodiscus anguste-lineata,Tha-

lassiosira polychorda)

Thalassiosira concaviuscula Makarova

Thalassiosira curviseriata Takano ${ }^{\mathrm{h}}$

Thalassiosira decipiens (Grunow) Jörgensen

Thalassiosira delicatula Ostenfeld

Thalassiosira diporocyclus Hasle

Thalassiosira eccentrica (Ehrenberg) Cleve

(syn. Coscinodiscus eccentricus)

Thalassiosira hendeyi Hasle et Fryxell

Thalassiosira cf kuschirensis Takano

Thalassiosira lundiana Fryxell

Thalassiosira mala Takano

Thalassiosira minima Gaarder

Thalassiosira monoporocyclus Hasle

Thalassiosira nordenskioeldii Cleve

Thalassiosira oceanica Hasle

Thalassiosira proschkinae Makarova

Thalassiosira punctigera (Castracane) Hasle (syn

Thalassiosira angstii, Thalassiosira japonica)

Thalassiosira rotula Meunier

Thalassiosira subtilis (Ostenfeld) Gran

Thalassiosira tealata Takano ${ }^{\mathrm{j}}$

Thalassiosira tenera Proshkina-Lavrenko

Triceratium favus Ehrenberg

Dinophyceae

Actiniscus pentasterias (Ehrenberg) Ehrenberg

(syn. Gymnaster pentasterias)

Akashiwo sanguinea (Hirasaki) Hansen et

Moestrup (syn. Gymnodinium sanguineum,

Gymnodinium splendens)

Amoebophrya ceratii (Koeppen) Cachon

Amylax triacantha (Jörgensen) Sournia

(syn. Gonyaulax triacantha)

Ceratium arietinum Cleve $(=$ Ceratium

bucephalum ${ }^{\mathrm{k}}$

Ceratium furca (Ehrenberg) Claparède

et Lachmann

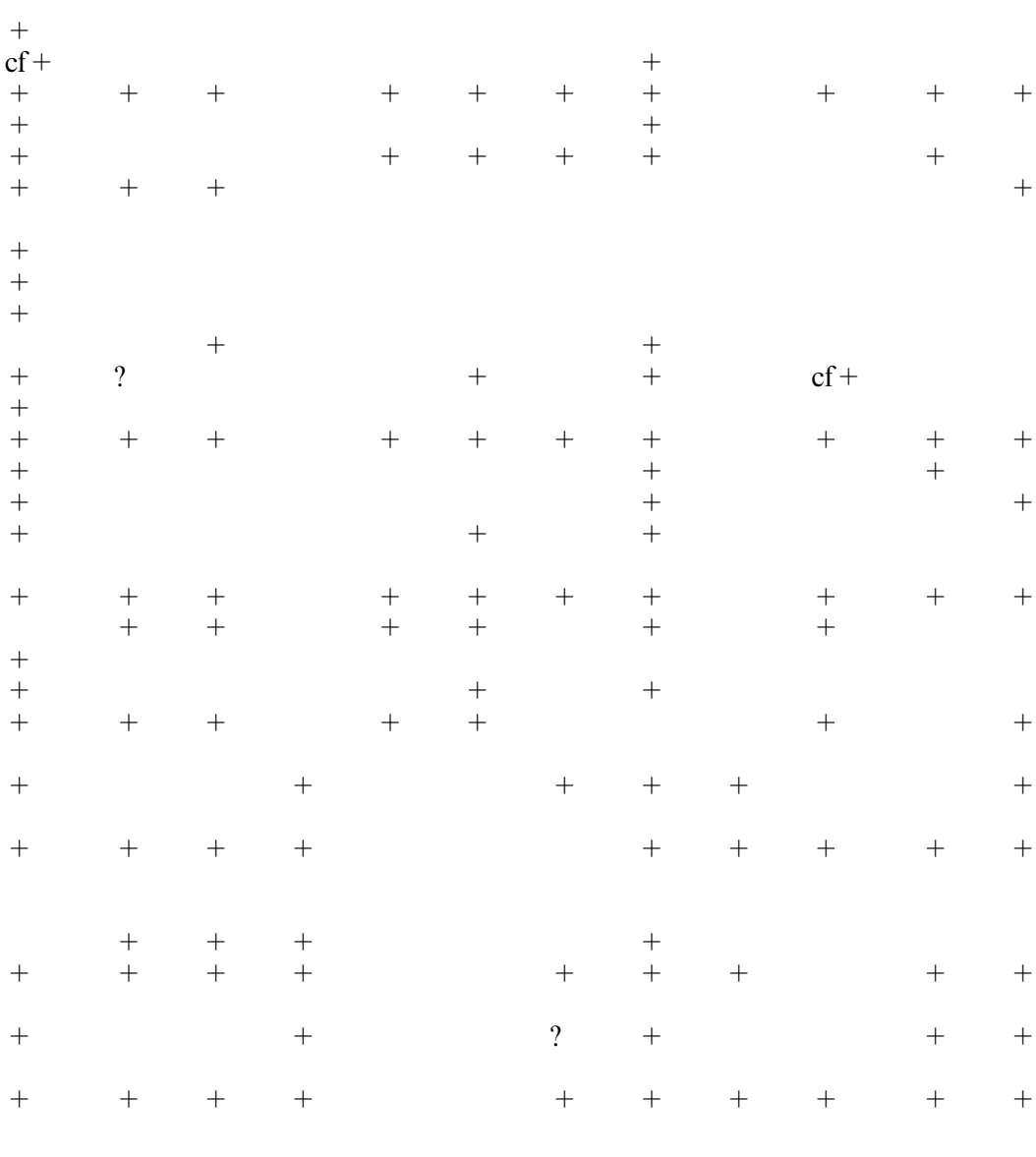


Table 1 (Contd.)

Species

Listed in:

\begin{tabular}{llllllllllll}
\hline 1 & 2 & 3 & 4 & 5 & 6 & 7 & 8 & 9 & 10 & 11 & 12
\end{tabular}

Ceratium fusus (Ehrenberg) Dujardin

Ceratium horridum (Cleve) Gran

(syn. Ceratium intermedium)

Ceratium lineatum (Ehrenberg) Cleve

Ceratium longipes (Bailey) Gran

Ceratium macroceros (Ehrenberg) Vanhöffen

Ceratium tripos (Müller) Nitzsch

Dinophysis acuminata Claparède et Lachmann $\mathbf{t}$

Dinophysis acuta Ehrenberg t

Dinophysis dens Pavillard

Dinophysis norvegica Claparède et Lachmann $\mathbf{t}$

Dinophysis rotundata Claparède et Lachmann

(syn. Phalacroma rotundatum)

Diplopelta bomba Stein ex Jörgensen (syn.

Dissodinium asymmetricum, Diplopsalisa

symmetrica) ${ }^{1}$

Diplopsalis lenticula Bergh

Dissodinium pseudocalani (Gönnert) Drebes

ex Elbrächter et Drebes

Dissodinium pseudolunula Swift ex Elbrächter

et Drebes

"Exuviaella marina" Cienkowski

Fragilidium subglobosum (v. Stosch) Balech

(syn. Helgolandinium subgobosum)

Gonyaulax diegensis Kofoid

Gonyaulax digitale (Pouchet) Kofoid

Gonyaulax spinifera (Claparède

et Lachmann) Diesing

Gonyaulax verior Sournia

Gymnodinium chlorophorum Elbrächter

et Schnepf (syn. Lepidodinium viride) ${ }^{\mathrm{m}}$

Gymnodinium cf endofasciculum Campell

Gymnodinium gracile Bergh (syn. Gymnodinium

abbreviatum, Gymnodinium lohmannii)

Gymnodinium lebourae Pavillard

Gymnodinium striatissimum Hulburt (syn.

Gymnodinium heterostriatum*)

Gyrodinium britannicum Kofoidet Swezy

Gyrodinium calyptoglyphe Lebour

(syn. Sclerodinium calyptoglyphe)

Gyrodinium lacryma (Meunier) Kofoid et Swezy ${ }^{\text {n }}$

Gyrodinium prunus (Wulff) Lebour ${ }^{\circ}$

Gyrodinium spirale (Bergh) Kofoid et Swezy

Gyrodinium undulans Hulburt

Heterocapsa minima Pomroy

Heterocapsa niei (Loeblich) Morill et Loeblich

(syn. Cachonina niei)

Heterocapsa rotundata (Lohmann) Hansen

(syn. Katodinium rotundatum, Amphidinium rotundatum)

Heterocapsa triquetra (Ehrenberg) Stein

(syn. Peridinium triquetrum)

Karenia mikimotoi (Miyake et Kominami ex Oda)

Hansen et Moestrup (syn. Gymnodinium

mikimotoi,Gyrodinium aureolum $\left.{ }^{*}\right)^{\mathrm{p}} \mathbf{t}$

Katodinium glaucum (Lebour) Loeblich

(syn. Massartia glauca)

Mesoporus perforatus (Gran) Lillick

Nematodinium armatum (Dogiel) Kofoid et Swezy

Noctiluca scintillans (Macartney) Kofoid

(syn. Noctiluca miliaris)

Oblea rotunda (Lebour) Balech ex Sournia (syn.

Peridiniopsis rotunda, Glenodinium rotundum)

Oodinium pouchetii (Lemmermann) Chatton

\begin{tabular}{|c|c|c|c|c|c|c|c|c|c|}
\hline+ & + & + & + & + & + & + & + & + & + \\
\hline+ & + & + & + & + & + & + & + & + & + \\
\hline+ & + & + & + & + & + & + & + & + & + \\
\hline+ & + & + & + & + & + & + & & + & + \\
\hline+ & + & + & + & + & + & & + & + & + \\
\hline+ & + & + & + & + & + & + & + & + & + \\
\hline+ & + & + & + & & + & + & + & + & + \\
\hline+ & + & + & + & + & + & + & + & + & + \\
\hline $\mathrm{cf}+$ & & & + & & + & + & & & + \\
\hline+ & + & + & + & + & + & + & & + & + \\
\hline+ & + & + & + & + & + & + & + & + & + \\
\hline+ & + & + & + & & + & + & + & & + \\
\hline+ & & & + & + & + & + & + & + & + \\
\hline+ & + & + & + & & & & & & \\
\hline+ & + & + & + & & + & & + & + & + \\
\hline+ & & & & & & & + & & \\
\hline+ & + & + & + & & + & + & & & + \\
\hline+ & & & + & & & & & & \\
\hline+ & + & + & + & + & + & + & + & + & + \\
\hline+ & + & + & + & + & + & + & + & + & + \\
\hline $\begin{array}{l}+ \\
+\end{array}$ & & & + & & + & + & & + & $\begin{array}{l}+ \\
+\end{array}$ \\
\hline+ & & & & & + & & & & + \\
\hline $\mathrm{cf}+$ & + & + & + & & + & + & & + & + \\
\hline+ & & & + & & + & + & + & & + \\
\hline+ & + & + & + & & + & & + & & + \\
\hline+ & + & + & + & & + & & + & & \\
\hline+ & + & + & + & & & + & + & & + \\
\hline$?+$ & $?+$ & & + & & + & & $\mathrm{cf}+$ & & \\
\hline$?+$ & + & + & + & & & + & & & \\
\hline+ & + & + & + & & + & + & + & + & + \\
\hline+ & & & + & + & + & + & & & + \\
\hline $\begin{array}{c}\mathrm{cf}+ \\
+\end{array}$ & & & + & & $\mathrm{cf}+$ & + & & & + \\
\hline & & & & & & & & & \\
\hline+ & & & + & + & + & + & + & + & + \\
\hline+ & + & + & + & + & + & + & + & + & + \\
\hline$?$ & + & + & + & + & + & + & & + & + \\
\hline+ & + & + & + & & + & + & + & & + \\
\hline+ & + & + & + & + & + & + & + & & + \\
\hline+ & + & + & + & & + & & & & \\
\hline+ & + & + & + & + & + & + & + & + & + \\
\hline+ & & & + & & + & + & + & + & + \\
\hline & + & + & + & & & & + & & \\
\hline
\end{tabular}


Table 1 (Contd.)

Species

Listed in:

\begin{tabular}{llllllllllll}
\hline 1 & 2 & 3 & 4 & 5 & 6 & 7 & 8 & 9 & 10 & 11 & 12 \\
\hline
\end{tabular}

Oxyrrhis marina Dujardin ${ }^{\mathrm{q}}$

Paulsenella chaetoceratis (Paulsen) Chatton

Polykrikos kofoidii Chatton (syn.

Polykrikos schwartzii*) ${ }^{\mathrm{r}}$

Polykrikos schwartzii Bütschli

Preperidinium meunierii (Pavillard) Elbrächter

(syn. Diplopeltopsis minor,Zygabikodinium

lenticulatum)

Prorocentrum balticum (Lohmann) Loeblich

(syn. Exuviaella balitica)

Prorocentrum micans Ehrenberg

Prorocentrum minimum (Pavillard) Schiller

(syn.Exuviaella mariae-lebouriae, Exuviaella apora* $^{*} \mathbf{t}$

Prorocentrum redfieldii Bursa (syn. Prorocentrum triestinum*) $\mathbf{t}$

Proterythropsis vigilans Marshall (syn.

Nematopsides vigilans)

Protoceratium reticulatum (Claparède et

Lachmann) Bütschli (syn. Gonyaulax grindleyi) t

Protoperidinium achromaticum (Levander) Balech

(syn. Peridinium achromaticum)

Protoperidinium bipes (Paulsen) Balech (syn.

Minuscula bipes, Peridinium minusculum)

Protoperidinium brevipes (Paulsen) Balech

(syn. Peridinium brevipes)

Protoperidinium cerasus (Paulsen) Balech

(syn. Peridinium cerasus) ${ }^{\mathrm{s}}$

Protoperidinium claudicans (Paulsen) Balech

(syn. Peridinium claudicans)

Protoperidinium conicum (Gran) Balech

(syn. Peridinium conicum)

Protoperidinium curtipes (Jörgensen) Balech

(syn.Peridinium curtipes)

Protoperidinium curvipes (Ostenfeld) Balech

(syn.Peridinium curvipes)

Protoperidinium denticulatum (Gran et Braarud)

Balech (syn. Peridinium denticulatum)

Protoperidinium depressum (Bailey) Balech

(syn. Peridinium depressum)

Protoperidinium divergens (Ehrenberg) Balech

(syn. Peridinium divergens) ${ }^{\mathrm{t}}$

Protoperidinium excentricum (Paulsen) Balech

(syn. Peridinium excentricum)

Protoperidinium granii (Ostenfeld) Balech

Protoperidinium leonis (Pavillard) Balech

(syn. Peridinium leonis)

Protoperidinium mariaelebouriae (Paulsen) Balech

(syn. Peridinium mariaelebouriae)

Protoperidinium minutum (Kofoid) Loeblich

(syn. Peridinium minutum)

Protoperidinium monospinum (Paulsen) Zonnefeld

et Dale

Protoperidinium oblongum (Aurivillius) Parke

et Dodge (syn. Peridinium oblongum)

Protoperidinium obtusum (Karsten) Parke

et Dodge (syn. Peridinium obtusum)

Protoperidinium ovatum Pouchet (syn. Peridinium ovatum)

Protoperidinium pallidum (Ostenfeld) Balech

(syn. Peridinium pallidum)

Protoperidinium pellucidum Bergh

(syn. Peridinium pellucidum)

Protoperidinium pentagonum (Gran) Balech

(syn. Peridinium pentagonum)

\begin{tabular}{|c|c|c|c|c|c|c|c|c|}
\hline+ & & & + & + & + & & + & + \\
\hline & + & + & + & & & & & \\
\hline+ & + & + & + & & + & & & \\
\hline+ & + & & + & + & + & + & + & + \\
\hline+ & + & + & + & & + & + & & + \\
\hline & + & + & + & + & + & + & + & + \\
\hline+ & + & + & + & + & + & + & + & + \\
\hline+ & & & + & & + & + & & + \\
\hline+ & & & + & & + & & + & \\
\hline+ & + & + & + & & + & + & & \\
\hline+ & + & + & + & + & + & + & + & + \\
\hline $\mathrm{cf}+$ & & & + & & & & + & + \\
\hline+ & + & + & + & + & + & + & + & + \\
\hline+ & + & + & + & + & + & + & + & + \\
\hline+ & $?+$ & & + & & + & & + & \\
\hline+ & + & + & + & & + & & + & + \\
\hline+ & + & + & + & + & + & + & + & + \\
\hline+ & + & + & + & & + & + & & \\
\hline+ & + & + & + & & + & & & + \\
\hline+ & + & + & + & & + & + & & \\
\hline+ & + & + & + & + & + & + & + & + \\
\hline $\mathrm{cf}+$ & $?+$ & & + & + & + & + & + & + \\
\hline+ & + & + & + & & + & + & + & + \\
\hline+ & & & + & + & + & + & + & + \\
\hline+ & & & + & & + & + & + & + \\
\hline+ & & + & + & + & + & & & \\
\hline+ & + & + & + & & + & & + & \\
\hline+ & & & $?$ & & & & & \\
\hline+ & & & + & & + & + & + & + \\
\hline+ & & & + & & & & & + \\
\hline+ & + & + & + & + & $\mathrm{cf}+$ & + & + & + \\
\hline+ & + & + & + & + & + & + & + & + \\
\hline+ & + & + & + & + & + & + & + & + \\
\hline+ & + & + & + & & + & + & + & + \\
\hline
\end{tabular}


Table 1 (Contd.)

\begin{tabular}{|c|c|c|c|c|c|c|c|c|c|c|c|c|}
\hline \multirow[t]{2}{*}{ Species } & \multicolumn{12}{|c|}{ Listed in: } \\
\hline & 1 & 2 & 3 & 4 & 5 & 6 & 7 & 8 & 9 & 10 & 11 & 12 \\
\hline $\begin{array}{l}\text { Protoperidinium punctulatum (Paulsen) Balech } \\
\text { (syn. Peridinium punctulatum) }\end{array}$ & $\mathrm{cf}+$ & & & + & & & & + & + & + & + & + \\
\hline $\begin{array}{l}\text { Protoperidinium pyriforme (Paulsen) Balech } \\
\text { (syn. Peridinium pyriforme) }\end{array}$ & + & + & + & + & & & + & + & & + & + & + \\
\hline $\begin{array}{l}\text { Protoperidinium steinii (Jörgensen) Balech } \\
\text { (syn. Peridinium steinii) }\end{array}$ & $\mathrm{cf}+$ & + & + & + & & & + & + & + & + & + & + \\
\hline $\begin{array}{l}\text { Protoperidinium subinerme (Paulsen) Loeblich } \\
\text { (syn. Peridinium subinerme) }\end{array}$ & + & + & + & + & & & + & + & + & + & & + \\
\hline $\begin{array}{l}\text { Protoperidinium thorianum (Paulsen) Balech } \\
\text { (syn. Peridinium thorianum) }\end{array}$ & + & + & + & + & & & + & + & + & + & & + \\
\hline Protoperidinium thulesense (Balech) Balech & $\mathrm{cf}+$ & & & + & & & & & & & & \\
\hline Pyrophacus horologium Stein & + & + & + & + & & & + & + & + & & + & + \\
\hline Scrippsiella lachrymosa Lewis & $\mathrm{cf}+$ & & & & & & & + & & & & \\
\hline $\begin{array}{l}\text { Scrippsiella trochoidea (Stein) Loeblich (syn. } \\
\text { Peridinium trochoideum, Scrippsiella faeroense) }\end{array}$ & + & + & + & + & & & & + & + & + & + & + \\
\hline $\begin{array}{l}\text { Spatulodinium pseudonoctiluca (Pouchet) Cachon } \\
\text { et Cachon ex Loeblich et Loeblich }\end{array}$ & + & & & + & & & & + & + & & & + \\
\hline Torodinium robustum Kofoid et Swezy & + & + & + & + & & & & + & + & + & & + \\
\hline Warnowia rosea (Pouchet) Kofoid et Swezy & $\mathrm{cf}+$ & & & + & & & & + & + & & & + \\
\hline
\end{tabular}

*Misidentification, not taxonomic synonymy

aAccording to Jensen and Moestrup (1998), Chaetoceros compressus is probably mistaken for Chaetoceros contortus

${ }^{b}$ According to Jensen and Moestrup (1998), Chaetoceros willei is a synonym of Chaetoceros affinis

${ }^{\mathrm{c} C o s c i n o d i s c u s ~ c e n t r a l i s E h r e n b e r g ~ i n ~ H a r t l e y ~(1986) ~}$

${ }^{\mathrm{d} C o s c i n o d i s c u s ~ p e r f o r a t u s v a r . ~ p a v i l l a r d i i ~ i n ~ H a r t l e y ~(1986) ~}$

${ }^{\mathrm{e}}$ Species introduced in 1977, Coscinodiscus nobilis in Robinson et al. (1980)

${ }^{\mathrm{f}}$ Species introduced in 1903, Odontella (as Biddulphia)sinensisin Ostenfeld (1908)

${ }^{\mathrm{g}}$ Rhizosolenia alata f.indica in Hartley (1986)

${ }^{\mathrm{h}}$ Possibly identical to Thalassios iratealata Takano

iSpecies introduced in 1978 (see Nehring1998; Reise et al.1999)

${ }^{\mathrm{j}}$ Species possibly introduced in 1950 (see Nehring1998; Reise et al.1999)

with a scanning electron microscope. For frustule preparation, diatom cultures were boiled in nitric acid and washed 10 times with distilled water.

The species are listed alphabetically within each class. Some synonyms, previously in common use, are given behind the species names for an easier comparison between the findings presented here and older publications and the cited lists.

\section{Results}

Table 1 gives a list of the marine phytoplankton diatoms and dinoflagellates found in Helgoland and other North Sea areas

The following changes should also be noted: the genus Neocalyptrella Hernández-Becerril et Meave has replaced Calyptrella (Hernández-Becerril and Meave del Castillo 1997); and Stauropsis membranacea is a synonym of Meuniera membranacea (Cleve) P.C. Silva (Hasle and Syvertsen 1997).

During the year of revision (May 2003 to April 2004), some additional species were recorded at the Helgoland
${ }^{\mathrm{k}}$ Ceratium bucephalum in Heimdal et al. (1973)

${ }^{1}$ Diplopsalopsis bomba in Kuylenstierna and Karlson (2000)

${ }^{\mathrm{m}}$ See Elbrächter and Schnepf (1996), Sournia et al. (1992); L. viride is a misidentification in some cases and not a real synonym

${ }^{\mathrm{n}}$ Requires re-investigation, see comments in Drebes and Elbrächter (1976, p. 81)

${ }^{\mathrm{o}}$ Requires re-investigation

possibly an introduced species (see Braarud and Heimdal1970; Hickel et al.1971; Elbrächter1999; Reise et al.1999)

${ }^{\mathrm{q}}$ Recorded in a tidal pool (sample from Dr S. Kühn), not at the Helgoland Reede station

${ }^{\mathrm{r}}$ In Drebes (1974) as Polykrikos schwartzii (p. 125, Figs. 106a, b)

${ }^{\mathrm{s}}$ May be in Drebes (1974) as Peridinium sp. (p. 134, Fig. 117c)

${ }^{\mathrm{t}}$ Requires re-investigation

uIn Drebes (1974) as Peridinium punctulatum (p. 140, Figs. 122a, b)

Reede station: Chaetoceros rostratus Lauder; Dactyliosolen fragilissimus (Bergon) Hasle; Fragilaria islandica Grunow sensu Drebes (1974); Stephanopyxis cf palmeriana (Greville) Grunow; and Amphidinium crassum Lohmann.

\section{Discussion}

One hundred and thirty-two species of diatoms from 53 genera and 95 species of dinoflagellates from 35 genera were recorded from net samples and cultures. From these 227 taxa, 35 diatoms and 28 dinoflagellate taxa were documented in the Helgoland phytoplankton for the first time. Eleven diatom and four dinoflagellate species could not be found again. Since the publication of the 1976 list (Drebes and Elbrächter 1976), numerous nomenclatural changes have occurred. This list does not claim to be complete, but provides an updated listing. Further continuous observations will be necessary to complete the species list.

Some taxa have been observed but not yet identified to species level, e.g. Thalassiosira spp, Chaetoceros spp, 
Cyclotella sp, Licmophora spp, Navicula spp, Nitzschia $\mathrm{sp}$, Pleurosigma spp, some benthic diatoms, dinoflagellate species of the Diplopsalis group, Gymnodinium spp, Gyrodinium spp, and Protoperidinium spp. In addition, Pseudo-nitzschia multiseries (Hasle) Hasle was identified in earlier Helgoland samples (Hasle 1995; Hasle et al. 1996) and should be mentioned here because it is toxic.

There have been some single records of benthic, sanddwelling dinoflagellates: Adenoides eludens (Herdman) Balech, 1956; Amphidinium bipes Herdman, 1924; Amphidinium glabrum Hoppenrath \& Okolodkov, 2000; Amphidiniopsis galericulata Hoppenrath, 2000; Prorocentrum clipeus Hoppenrath, 2000; and Thecadinium ornatum Hoppenrath, 2000. In my opinion, these tychoplanktonic species occurred only "by accident" in the plankton and therefore they were not included in the present checklist. Other regularly observed plankton species from other groups include: Phaeocystis globosa Scherffel; Dictyocha speculum Ehrenberg; and Ebria tripartita (Schumann) Lemmermann (see also Drebes 1974).

Detection of long-term trends in the species composition needs more detailed investigations. The high number of "first" records (63) should not be interpreted as a real change in species composition but as a result of more detailed species knowledge. Drebes and Elbrächter have experienced the same results at the Sylt station, Wadden Sea since 1976. Additionally, there have been some newly described species since the 1970s. Some small Thalassiosira species could only be identified using scanning electron microscopy. Since Drebes and Elbrächter's (1976) checklist, only Coscinodiscus wailesii (Boalch and Harbour 1977; Robinson et al. 1980) and Thalassiosira punctigera (Nehring 1998) have been documented as introduced species. The present list may include some native species which were only discovered recently but may have been around long before without being noticed, because our attention and taxonomic expertise generally decrease with decreasing size of the organisms. Therefore, there is a great need for intensive phytoplankton species documentation as a baseline for a less ambiguous recognition of changes in the species composition with respect to toxic species occurrence and global change aspects. A continuous routine net phytoplankton species monitoring, in combination with the regular determination of the species abundance, is essential.

The comparison of checklists is problematic because of the subjective taxon recognition by the authors and the lack of illustrations of the observed specimens. But it may be useful (e.g. for biogeographic comparisons) to compare this species list with data from other areas, and for convenience records have been added to Table 1 (columns labelled 4-12; Grøntved 1952; Braarud et al. 1953).

Acknowledgements I would like to thank H. Halliger, Wadden Sea station Sylt, AWI, for the introduction to phytoplankton diatom identification; M. Elbrächter, Taxonomische Arbeitsgruppe am Forschungsintitut Senckenberg, for his taxonomic advice and his help in the identification of some dinoflagellates; and R. Crawford,
AWI Bremerhaven, for valuable comments and his kindness in correcting the English. L. Medlin, AWI Bremerhaven, kindly helped identify the Pseudo-nitzschia species and J.E.B. Rines, University of Rhode Island, identified Chaetoceros rostratus.

\section{References}

Boalch GT, Harbour DS (1977) Unusual diatom off the coast of south-west England and its effect on fishing. Nature 269:687688

Braarud T, Heimdal BR (1970) Brown water on the Norwegian coast in autumn 1966. Nytt Mag Bot 17:91-97

Braarud T, Gaarder KR, Grøntved J (1953) The phytoplankton of the North Sea and adjacent waters in May 1948. Rapp P-V Réun Conseil Int Explor Mer 133:1-87

Drebes G (1974) Marines Phytoplankton. Eine Auswahl der Helgoländer Planktonalgen (Diatomeen, Peridineen). Thieme, Stuttgart.

Drebes G, Elbrächter M (1976) A checklist of planktonic diatoms and dinoflagellates from Helgoland and List (Sylt), German Bight. Bot Mar 19:75-83

Elbrächter M (1999) Exotic flagellates of coastal North Sea waters. Helgol Meeresunters 52:235-242

Elbrächter M, Schnepf E (1996) Gymnodinium chlorophorum, a new, green, bloom-forming dinoflagellate (Gymnodiniales, Dinophyceae) with a vestigial prasinophyte endosymbiont. Phycologia 35:381-393

Grøntved J (1952) Investigations on the phytoplankton in the southern North Sea in May 1947. Medd Komm Dan Fisk Havunders Ser Plankt 5:1-49

Hagmeier E, Spindler H, Van Beusekom J, Wiltshire K (2004) Historical background to the timeseries. Helgol Mar Res (in press)

Hällfors G Baltic Sea phytoplankton species checklist. http://jolly.fimr.fi/Checklist3.nsf

Hansen G, Larsen J (1992) Dinoflagellater i danske farvande. In: Thomsen HA (ed) Plankton i de indre danske farvande. Havforskning fra Miljostyrelsen 11, pp 45-155

Harms J (1993) Check list of species (algae, invertebrates and vertebrates) found in the vicinity of the island of Helgoland (North Sea, German Bight) - a review of recent records. Helgol Meeresunters 47:1-34

Hartley B (1986) A check-list of the freshwater, brackish and marine diatoms of the British Isles and adjoining coastal waters. J Mar Biol Assoc UK 66:531-610

Hasle GR (1995) Pseudo-nitzschia pungens and P. multiseries (Bacillariophyceae): nomenclatural history, morphology, and distribution. J Phycol 31:428-435

Hasle GR, Syvertsen EE (1997) Marine diatoms. In: Tomas CR (ed) Identifying marine phytoplankton. Academic Press, pp 5385

Hasle GR, Lange CB, Syvertsen EE (1996) A review of Pseudonitzschia, with special reference to the Skagerrak, North Atlantic, and adjacent waters. Helgol Meeresunters 50:131-175

Heimdal BR, Hasle GR, Throndsen J (1973) An annotated checklist of plankton algae from the Oslofjord, Norway (1951-1972). Norw J Bot 20:13-19

Hendey NI (1974) A revised check-list of British marine diatoms. J Mar Biol Assoc UK 54:277-300

Hernández-Becerril DU, Meave del Castillo ME (1997) Neocalyprella gen. nov., a new name to replace Calyprella HernándezBecerril et Meave. Phycologia 36:329

Hickel W, Hagmeier E, Drebes G (1971) Gymnodinium blooms in the Helgoland Bight (North Sea) during August, 1968. Helgol Wiss Meeresunters 22:401-416

Jensen KJ, Moestrup Ø (1998) The genus Chaetoceros (Bacillariophyceae) in inner Danish coastal waters. Opera Bot 133:1-68

Kuylenstierna M, Karlson B (2000) Checklist of phytoplankton in Skagerrak-Kattegat. http://www.marbot.gu.se/SSS/SSShome.htm 
Leewis RJ (1985) Phytoplankton off the Dutch coast. A base line study on the temporal and spatial distribution of species in 1974 and 1975. PhD Thesis, Van Gorcum, Assen

Nehring S (1998) Non-indigenous phytoplankton species in the North Sea: supposed region of origin and possible transport vector. Arch Fish Mar Res 46:181-194

Ostenfeld CH (1908) On the immigration of Biddulphia sinensis Grev. and its occurrence in the North Sea during 1903-1907. Medd Komm Havunders Ser Plankt 1:1-44

Pankow H (1990) Ostsee-Algenflora. Fischer, Jena

Parke M, Dixon PS (1976) Check-list of British marine algae - 3rd revision. J Mar Biol Assoc UK 56:527-594

Reise K, Gollasch S, Wolff WJ (1999) Introduced marine species of the North Sea coasts. Helgol Meeresunters 52:219-234

Robinson GA, Budd TD, John AWG, Reid PC (1980) Coscinodiscus nobilis (Grunow) in continuous plankton records, 197778. J Mar Biol Assoc UK 60:675-680
Sournia A, Belin C, Billard C, Catherine M, Erard-Le Denn E, Fresnel J, Lassus P, Pastoureaud A, Soulard R (1992) The repetitive and expanding occurrence of a green, bloom-forming dinoflagellate (Dinophyceae) on the coasts of France. Cryptogam Algol 13:1-13

Van Beusekom J, Mangelsdorf P, Wiltshire K (2004) The nutrient data of the Helgoland Reede: quality assessment and usefulness. Helgol Mar Res (in press)

Wiltshire K, Dürselen C (2004) The Phytoplankton data of the Helgoland Reede: quality assessment and usefulness. Helgol Mar Res (in press)

Wiltshire K, Dürselen C, Spindler H (2004) Description of the Helgoland data sets and archiving. Helgol Mar Res (in press) 